\title{
Formulation and pharmacokinetic evaluation of rifampicin solid lipid nanoparticles
}

\author{
Ahmed GARDOUH ${ }^{1,2} * \mathbb{D}$, Alshimaa GAMAL ${ }^{3}(\mathbb{D})$, Shadeed GAD ${ }^{1}(\mathbb{D}$ \\ 1 Department of Pharmaceutics and Industrial Pharmacy, Faculty of Pharmacy, Suez Canal University, \\ Ismailia41511, Egypt. \\ 2 Department of Pharmaceutical sciences, Faculty of Pharmacy, Jadara University, Irbid 22110, Jordan. \\ 3 Department of Pharmaceutics and Industrial Pharmacy, Faculty of Pharmacy, Sinai University, Ismailia 41511, \\ Egypt. \\ * Corresponding Author. E-mail: ahmed_mahmoud@pharm.suez.edu.eg (A.G.); Tel. +20-101-379 0828.
}

Received: 17 February 2020 / Revised: 13 May 2020/ Accepted: 29 May 2020

\begin{abstract}
This study aims to optimize RIF loaded solid lipid nanoparticles (RIF-SLNs) to sustain its release and enhance its oral absorption and bioavailability. RIF loaded SLNs were formulated by a modified micro emulsionbased technique using two different lipids (Cetyl palmitate and Glyceryl monostearate) and two different surfactants (Tween ${ }^{\circledR 80}$ and Poloxamer 188). Particle size, polydispersity index (PDI), zeta potential, entrapment efficiency (E.E), drug loading capacity (L.C), in vitro drug release, differential scanning calorimetry (DSC), Fourier transform infrared spectroscopy (FTIR) and transmission electron microscopy (TEM) were determined for RIF loaded SLNs formulae. Pharmacokinetic study was performed on optimized RIF-SLNs, marketed RIF and pure drug suspension in Wistar rats. The particle size, PDI, E.E\% and L.C\% of optimized formula were recorded as $0.183 \mu \mathrm{m}, 0.420,-34.7 \mathrm{mV}, 80.8 \%$ and $0.216 \%$, respectively. In vitro release studies suggested that all SLNs formulae possessed a burst release created from the unloaded drug and adsorbed drug molecules at SLNs surface then sustained release due to diffusion of drug from lipid matrix over a period of $120 \mathrm{~h}$. From the release kinetics data, the release rate of RIF from all formulae fitted into Higuchi's diffusion model. Pharmacokinetic study showed significant enhancement in RIF-SLNs relative bioavailability 5.86 and 2.33 folds in comparison with pure RIF suspension and marketed RIF. RIF loaded SLNs were formulated successfully by a modified micro emulsion-based method. Also, oral drug delivery can be enhanced by SLNs which showed improvement in the oral bioavailability of the drug.
\end{abstract}

KEYWORDS: Pharmacokinetic enhancement; rifampicin; In vitro release; solid lipid nanoparticles; glyceryl monostearate; cetyl palmitate.

\section{INTRODUCTION}

Solid lipid nanoparticles (SLNs) are colloidal lipid carriers, which are consisted of biodegradable lipids like hard fats, monoglycerides, highly purified triglycerides, complex glyceride mixtures or waxes that are solid at $25^{\circ} \mathrm{C}$. Unlike liposomes and emulsions, the particle-matrix of SLNs is composed of solid lipids [1]. There are several advantages for using of SLNs in drug delivery such as the possibility of controlling drug release and targeting, the ability of accommodation of both lipophilic and hydrophilic drugs, low toxicity and good biocompatibility, avoiding organic solvents incorporation in the production cycle and large-scale production. Oral administration of SLNs could increase the lymphatic transport of drugs, which decreases first-pass hepatic metabolism and increases oral bioavailability of the medicament [1,2]. Various methods are available for the preparation of SLNs such as hot high-pressure homogenization, cold highpressure homogenization, high shear homogenization followed by ultrasonication, solvent emulsification/evaporation process, and micro emulsion-based technique. The principle of this latter method is to disperse a warm $\mathrm{O} / \mathrm{W}$ microemulsion into a large amount of cold water forming SLNs. This method is easily processed at laboratory scale as microemulsion, the key intermediate product of this preparation would be formed spontaneously [3]. The removal process of excess water is difficult, so that some modifications are applied to the method to decrease the dispersion ratio to $1: 2$ and improve the stability of the prepared SLNs [4].

Tuberculosis (TB) is one of the most common and fatal diseases. Not only it is largely widespread in developing and underdeveloped countries but also, it is one of the major concerns among developed

How to cite this article: Gardouh A, Gamal Al, Gad S. Formulation and pharmacokinetic evaluation of rifampicin solid lipid nanoparticles. J Res Pharm. 2020; 24(4): 539-551. 
countries [4]. Rifampicin (RIF) is a strong first-line anti-tubercular drug (ATD) and regards effective in the treatment regimen of tuberculosis and leprosis. It is a bactericidal antibiotic, which inhibits the RNA synthesis process initiation through binding to DNA-dependent RNA polymerase. It is a broad-spectrum antibiotic, commonly used in the tuberculosis treatment regimen. RIF suffers from some drawbacks such as poor and erratic bioavailability, short half-life and high hepatotoxicity, resulting in sub-therapeutic levels of drug in the blood and elevated risk of development of multi-drug resistant tuberculosis [5]. The administration of RIF is recommended to be taken at empty stomach because food decreases the absorption of RIF [6]. The quality of antibacterial treatment can be enhanced by the use of nanoparticulate drug delivery systems through decreasing side effects, prolonging drug residence time and reducing the frequency of dosing.

This study aims to verify the enhancement of the oral absorption and bioavailability of rifampicin through developing rifampicin loaded solid lipid nanoparticles using a modified micro emulsion-based technique. Also, The effect of some formulation variables (lipid type and concentration) on particle size, zeta potential, polydispersity index, drug entrapment efficiency, drug loading, in vitro release, Differential Scanning Calorimetry, Fourier transform infrared spectroscopy, Transmission Electron Microscopy study and pharmacokinetic study will be investigated.

\section{RESULTS AND DISCUSSION}

\subsection{Preparation of RIF Loaded SLNs}

Nine different RIF loaded SLNs formulae produced by modified micro emulsion-based technique are presented in Table 1. Two types of lipids Cetyl palmitate (CP) and Glyceryl monostearate (GMS) were used at three different concentrations $(1 \%, 5 \%$ and $10 \% \mathrm{w} / \mathrm{w})$. Tween ${ }^{\circledR} 80$ at concentration $6 \% \mathrm{w} / \mathrm{w}$ was used as a surfactant and Poloxamer 188 at concentration $1 \% \mathrm{w} / \mathrm{v}$ was used as a co-surfactant. Based on rank order performed for all loaded RIF SLNs formulae, the best formula was chosen for DSC, FTIR, TEM and pharmacokinetic study.

Table 1. Characterizations of RIF loaded SLNs (mean $\pm S D, N=3$ ).

\begin{tabular}{lccccccc}
\hline Formulae & $\begin{array}{c}\text { Lipid } \\
\text { concentration } \\
\text { \% w/w }\end{array}$ & $\begin{array}{c}\text { CP : } \\
\text { GMS }\end{array}$ & $\begin{array}{c}\text { Particle } \\
\text { size }(\boldsymbol{\mu m})\end{array}$ & PDI & $\begin{array}{c}\text { Z potential } \\
(\boldsymbol{\zeta}) \\
(\mathbf{m V})\end{array}$ & $\begin{array}{c}\text { E.E } \\
\mathbf{( \% )}\end{array}$ & $\begin{array}{c}\text { L.C } \\
(\mathbf{r a t i o})\end{array}$ \\
\hline SLN-1 & 1 & $1: 3$ & $0.166 \pm 0.011$ & $0.282 \pm 0.040$ & $-24.9 \pm 0.400$ & $57.7 \pm 0.666$ & $0.154 \pm 0.002$ \\
SLN-2 & 1 & $1: 2$ & $0.172 \pm 0.017$ & $0.295 \pm 0.031$ & $-22.5 \pm 0.115$ & $60.3 \pm 0.656$ & $0.161 \pm 0.002$ \\
SLN-3 & 1 & $1: 1$ & $0.291 \pm 0.032$ & $0.243 \pm 0.042$ & $-21.0 \pm 0.551$ & $62.0 \pm 0.416$ & $0.165 \pm 0.001$ \\
SLN-4 & 5 & $1: 3$ & $0.183 \pm 0.012$ & $0.420 \pm 0.016$ & $-34.7 \pm 0.700$ & $80.8 \pm 0.400$ & $0.216 \pm 0.001$ \\
SLN-5 & 5 & $1: 2$ & $0.231 \pm 0.020$ & $0.430 \pm 0.022$ & $-31.9 \pm 0.666$ & $82.0 \pm 0.416$ & $0.219 \pm 0.001$ \\
SLN-6 & 5 & $1: 1$ & $0.372 \pm 0.108$ & $0.381 \pm 0.038$ & $-30.8 \pm 0.306$ & $83.3 \pm 0.404$ & $0.222 \pm 0.001$ \\
SLN-7 & 10 & $1: 3$ & $0.223 \pm 0.069$ & $0.591 \pm 0.033$ & $-41.2 \pm 0.416$ & $72.1 \pm 0.300$ & $0.192 \pm 0.001$ \\
SLN-8 & 10 & $1: 2$ & $0.390 \pm 0.103$ & $0.645 \pm 0.026$ & $-39.1 \pm 0.569$ & $74.2 \pm 0.833$ & $0.198 \pm 0.002$ \\
SLN-9 & 10 & $1: 1$ & $0.439 \pm 0.128$ & $0.619 \pm 0.052$ & $-37.6 \pm 0.351$ & $76.7 \pm 0.721$ & $0.205 \pm 0.002$ \\
\hline
\end{tabular}

\subsection{Measurement of zeta size, polydispersity index and zeta potential of loaded SLNs}

Polydispersity index (PDI) indicates the strength of particle size distribution. Values closer to zero indicate a homogeneous dispersion. PDI values less than 0.3 ensure homogeneity and uniformity of the drug in the system [7]. Table 1 and Figure 1 shows that particle size values are ranging from $0.166 \pm 0.011 \mu \mathrm{m}$ to $0.439 \pm 0.128 \mu \mathrm{m}$ and PDI values are ranging from $0.243 \pm 0.042$ to $0.645 \pm 0.026$. Increased lipid content over 5$10 \%$ resulted in increased particle size and PDI values. The reason is that increased dispersed phase viscosity resulted in decreased homogenization efficiency and increased particle agglomeration rate. The same observation was reported by Shah et al. on their study on rivastigmine loaded solid lipid nanoparticles [8].

Table 1 and Figure 2 shows the measured zeta potential of SLNs. As shown, all formulae were negatively charged, zeta potential ranged from $-21.0 \mathrm{mV}$ to $-41.5 \mathrm{mV}$. Higher zeta potential leads to an increase in the physical stability of the system due to the increase in the electrostatic repulsion force between the particles. Values higher than $+30 \mathrm{mV}$ and lower than $-30 \mathrm{mV}$, indicates good storage stability [9-13]. 
Zeta potential of SLNs containing an elevated concentration of Glyceryl monostearate was more negative than Cetyl palmitate SLNs. This result agrees with that obtained by previous studies [10, 14, 15]. The reason is that Glyceryl monostearate is an ester of fatty acid which increased the particle surface negative charge [16].

It was determined that as the lipid concentration increased, the zeta potential negative charge increased. This is because of the increase in lipid concentration would increase the drug loading as a negative charge on the drug would increase the surface negative charge of SLNs and formed SLNs with more negative zeta potential. Khalil et al. reported a similar observation [17].
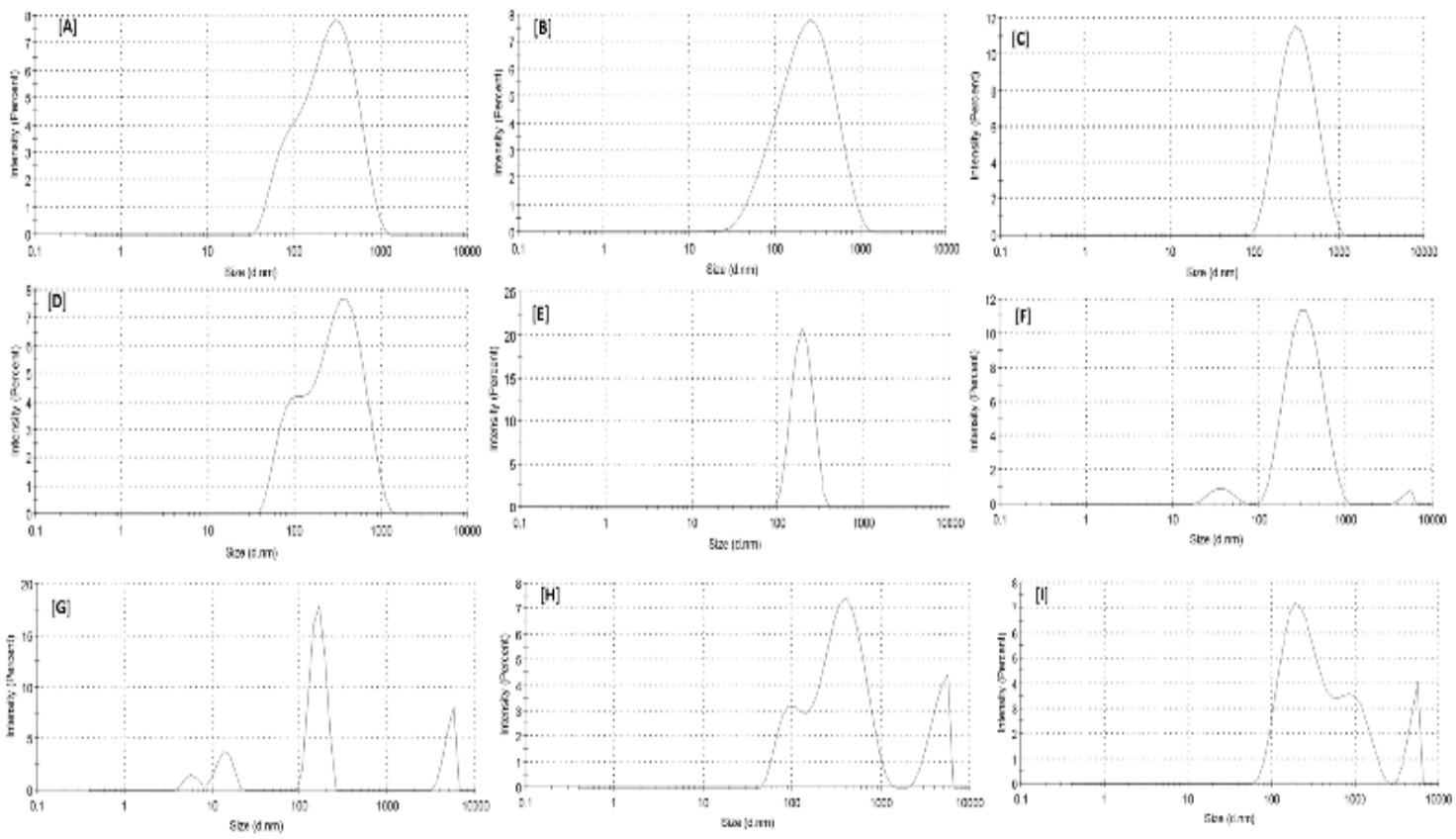

Figure 1. Particle size of (A) SLN-1, (B) SLN-2, (C) SLN-3, (D) SLN-4, (E) SLN-5, (F) SLN-6, (G) SLN-7, (H) SLN-8 and (I) SLN-9.
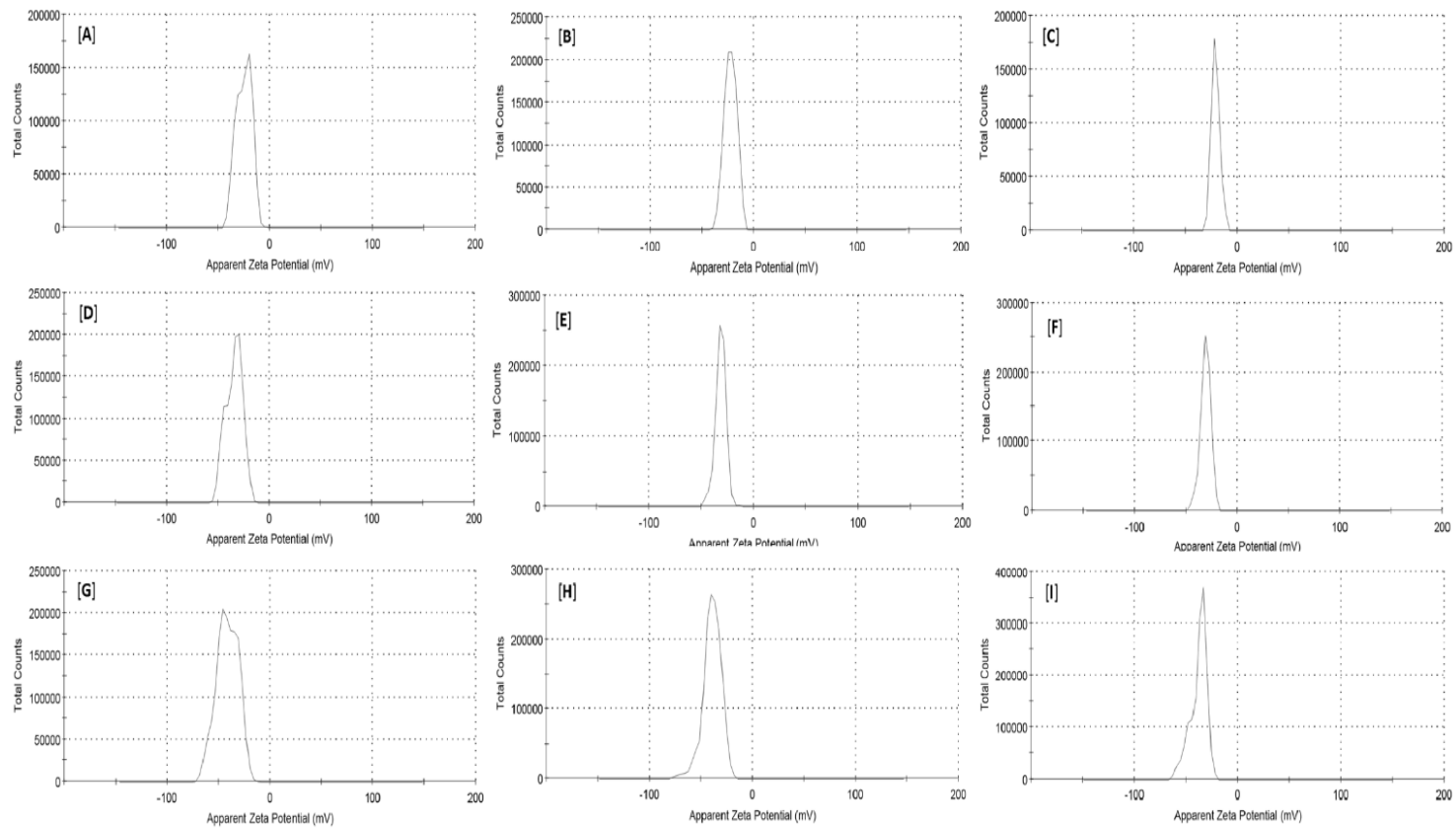

Figure 2. Zeta potential of (A) SLN-1, (B) SLN-2, (C) SLN-3, (D) SLN-4, (E) SLN-5, (F) SLN-6, (G) SLN-7, (H) SLN-8 and (I) SLN-9. 


\subsection{Encapsulation efficiency, loading capacity}

Table 1 reveals that the encapsulation efficiency and drug loading of SLNs with a high concentration of Cetyl palmitate are higher than Glyceryl monostearate SLNs. This is because of the variance in the structure of the two lipids. A lipid with high hydrophobicity (Cetyl palmitate) due to the attached long-chain fatty acid resulting in enhanced incorporation of lipophilic drugs such as rifampicin $[11,14,15]$.

Encapsulation efficiency and drug loading enhanced by increasing the lipid concentration from $1 \%$ to $5 \%$ but decreased upon further increase of the lipid to $10 \%$. The reason is that the tendency of the aggregates to coalesce at high concentrations of lipid. Also, the increase in the viscosity at the interface resulted in a decrease in solvent diffusion and smaller amount of lipid molecules would be passed into the aqueous phase. Therefore, the stability of the small aggregates at this lipid concentration decreased. Khalil et al. reported a similar observation on meloxicam SLNs [17].

\subsection{In vitro drug release studies}

Cumulative release profiles of RIF loaded SLNs formulae over 120-h time period are revealed in Figure 3. Profiles showed biphasic patterns, a burst release initiated by the unloaded drug and adsorbed drug molecules at SLNs surface followed by a slow release due to the diffusion of drug from lipid matrix [4, 18]. Fast release could be due to the enlarged surface area, elevated diffusion coefficient due to small particle size, small diffusion distance for the drug and low matrix viscosity [19].

The release rate of the drug was affected by the lipid matrix nature, concentration of surfactant and preparation parameters [20]. Surfactant concentration was optimized at $6 \%$, so the release of drug was affected by the type and quantity of the lipid matrix. It was investigated that lipid with high Glyceryl monostearate content showed highest release efficiency and lowest $t 1 / 2$ while in case of Cetyl palmitate the slow release and higher t1/2 might be due to the wax SLNs had long-term stability with good drug encapsulation efficiency. The long hydrophobic chain fatty acids of Cetyl palmitate highly retained lipophilic drugs resulting in sustained drug release [14].

Furthermore, the smaller particle size of GMS-SLNs resulted in increasing release efficiency. Difference in release data because of the particle size was observed by Mohyeldin et al. They suggested that a smaller size of nanoparticles might decrease the diffusion layer thickness between the surface and bulk lipid leading to fast release [21]. The lower melting point of GMS could lead to higher mobility at the analysis temperature. This observation was also suggested by Paolicelli et al [22].

It was observed that increasing the lipid concentration of SLNs from 1, 5 to $10 \%$ resulted in decreasing the release efficiency and increasing the $\mathrm{t} 1 / 2$ value. The reason is that enhanced encapsulation efficiency of the drug led to reducing drug partitioning at SLNs surface [17].

The release profile of all RIF loaded SLNs formulae are best fit to Higuchi's model, which describes the drug diffusion from matrix system. Excluding the burst release of early time data points, linear fits were recorded indicating the release of drug was through diffusion. These results were in identity with many studies which reported that the rate of drug release from SLNs followed Higuchi's diffusion model [7, 11, 18].

\subsection{Differential scanning calorimetry (DSC)}

Differential scanning calorimetry thermograms were measured to study crystallinity state as well as thermal characteristics of RIF. Crystallinity state of RIF could predict the physicochemical characteristics of the SLNs such as dissolution, saturation solubility and physical stability. Thermograms of pure RIF, Cetyl palmitate, Glyceryl monostearate, their physical mixture and RIF loaded SLNs (F4) are obtained in Figure 4. The melting point of Cetyl palmitate and Glyceryl monostearate is about $60^{\circ} \mathrm{C}$ and $54^{\circ} \mathrm{C}$ respectively, which were noticeable at bulk lipid thermograms. RIF thermogram showed characteristic endothermic and exothermic peaks at $183.17^{\circ} \mathrm{C}$ and $254.81^{\circ} \mathrm{C}$ respectively.

The endothermic peak of RIF was shifted to a lower temperature in the physical mixture thermogram because the melting lipids tend to solubilize RIF. This result was previously observed by Ghaderkhani et al. after the disappearance of the drug melting peak in the physical mixture thermogram of RIF and Stearic acid for SLNs preparation [23]. 
The thermogram of RIF loaded SLNs (F4) showed both endothermic and exothermic peak. The endothermic peak indicated the lipid melting point which was shifted to a temperature lower than that of bulk lipid and physical mixture, this is because of the nanosize of SLNs, high surface area and presence of the surfactant, i.e., the depression could be attributed to Kelvin effect [7]. Kelvin reported that small isolated particles had a melting temperature lower than bulk materials.

The exothermic peak was shifted to a lower temperature of $240^{\circ} \mathrm{C}$ compared to pure RIF thermogram. This observation was reported in a previous study where the peak of RIF in nanosuspension was shifted to a lower temperature. They attributed this observation to the partial transformation of RIF from the crystalline to amorphous form resulting in enhanced solubility [21].

Disappearance of RIF endothermic peak in RIF loaded SLNs (F4) thermogram indicated its incorporation within SLNs, similar results were obtained in a previous study [24].

Physical mixture $(\Delta \mathrm{H}=49.37 \mathrm{~J} / \mathrm{g})$ and RIF loaded SLNs $(\mathrm{F} 4)(\Delta \mathrm{H}=51.32 \mathrm{~J} / \mathrm{g})$ showed a decrease in the enthalpy value of RIF than pure RIF $(\Delta \mathrm{H}=87.18 \mathrm{~J} / \mathrm{g})$. This observation could be explained by the development of less order crystals which released a smaller amount of energy than crystalline ones [17]. All previous results indicated excellent entrapment of RIF within SLNs core.

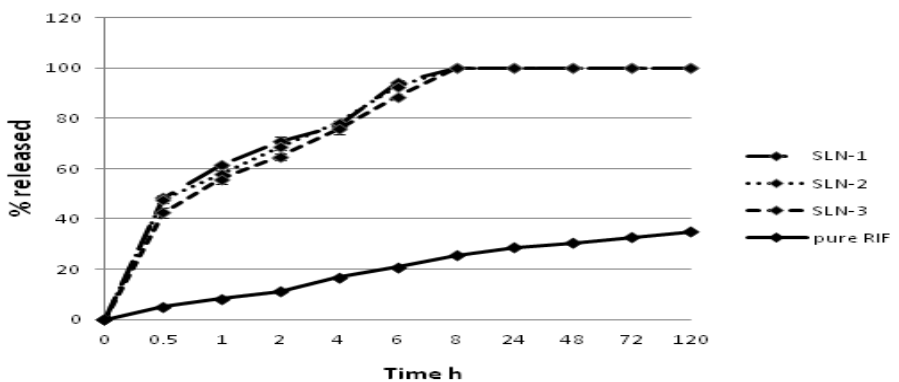

(a)

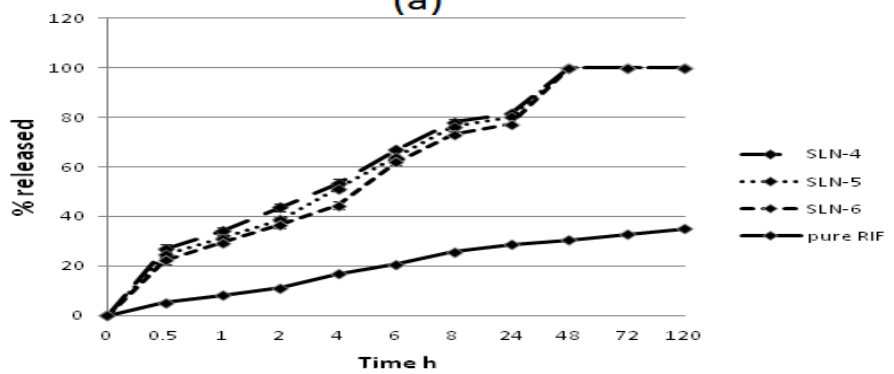

(b)

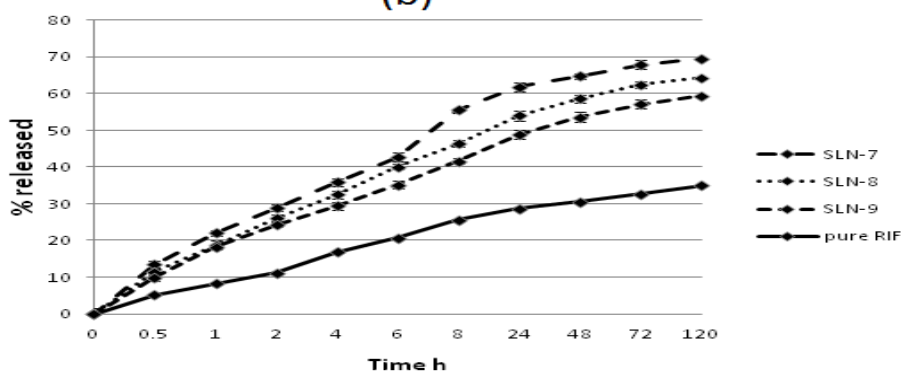

(c)

Figure 3. In vitro release profiles RIF loaded SLNs formulae compared with pure RIF: (a), at lipid concentration 1\%; (b), at lipid concentration 5\%; (c), at lipid concentration 10\%. Data were expressed as the mean \pm S.E. $(n=3)$. 

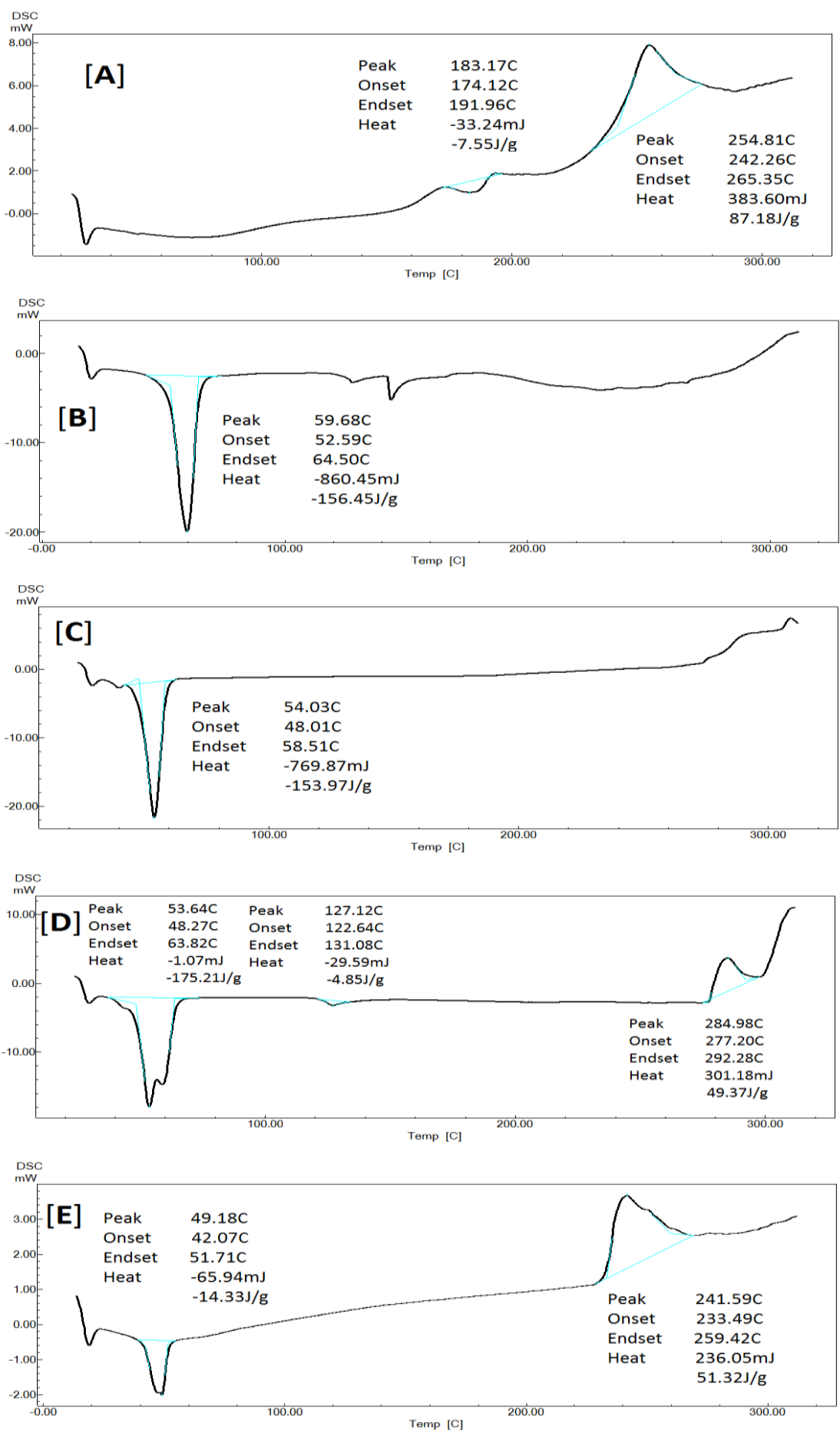

Figure 4. DSC thermograms of $[\mathrm{A}]$ pure RIF, [B] cetyl palmitate, [C] glyceryl monostearate, [D] physical mixture and [E] RIF loaded SLNs (F4).

\subsection{Fourier transform infrared spectroscopy (FTIR)}

Infrared spectra of RIF, Cetyl palmitate, Glyceryl monostearate, their physical mixture and RIF loaded SLNs (F4) are investigated at Figure 5. FTIR spectrum of RIF showed characteristic absorption bands at $3430.74 \mathrm{~cm}^{-1}(-\mathrm{OH}), 2922.59 \mathrm{~cm}^{-1}(-\mathrm{C}=\mathrm{O}), 1641.13 \mathrm{~cm}^{-1}(-\mathrm{CONH} 2), 1456.96 \mathrm{~cm}^{-1}(-\mathrm{C}=\mathrm{C}-)$ stretching and $1244.83 \mathrm{~cm}^{-1}(-\mathrm{C}-\mathrm{O}-\mathrm{C}-)$. Also, the IR spectrum of Cetyl palmitate showed absorption bands at $2919.7 \mathrm{~cm}^{-1}(-$ $\mathrm{C}-\mathrm{H})$ aliphatic, $1732.73 \mathrm{~cm}^{-1}(-\mathrm{C}=\mathrm{O})$ and $1184.08 \mathrm{~cm}^{-1}(-\mathrm{C}-\mathrm{O}-\mathrm{C}-)$. The IR spectrum of Glyceryl monostearate showed absorption bands at $3391.21 \mathrm{~cm}^{-1}(-\mathrm{OH}), 2919.7 \mathrm{~cm}^{-1}(-\mathrm{C}-\mathrm{H})$ aliphatic, $1733.69 \mathrm{~cm}^{-1}(-\mathrm{C}=\mathrm{O})$ and $1184.08 \mathrm{~cm}^{-1}(-\mathrm{C}-\mathrm{O}-\mathrm{C}-)$. 
All characteristic absorption bands of RIF present in their physical mixture and RIF loaded SLNs (F4) spectra indicated no modification in the chemical structure of RIF. These results agreed with that obtained by Rahman et al., who prepared risperidone SLNs and found that FTIR data showed no physicochemical interaction between drug and lipid [25].
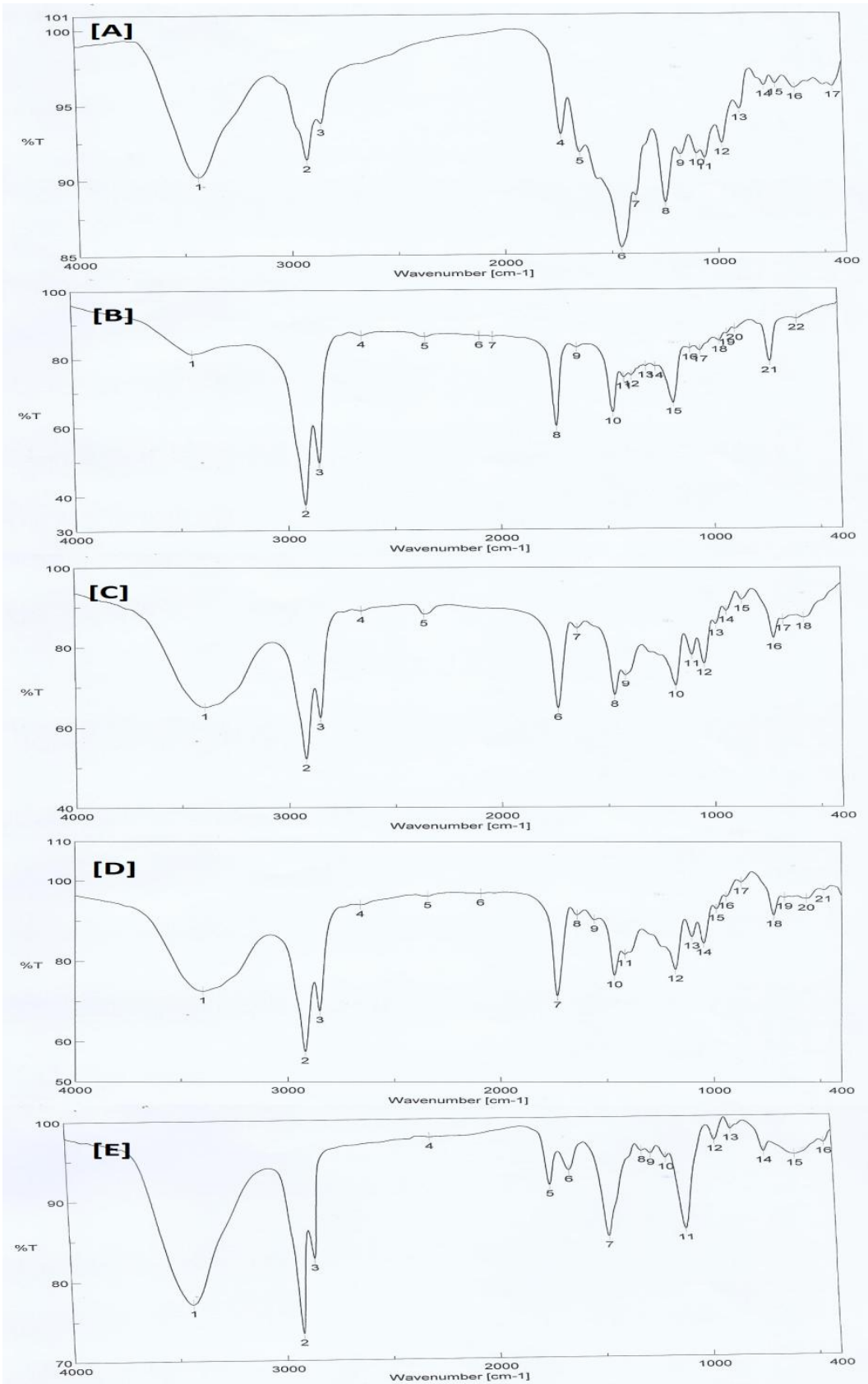

Figure 5. FTIR spectra of $[\mathrm{A}]$ pure RIF, [B] cetyl palmitate, [C] glyceryl monostearate, [D] their physical mixture and [E] RIF loaded SLNs (F4).

\subsection{Transmission electron microscopy (TEM)}

Morphology of RIF loaded SLNs (F4) was assessed using TEM as revealed in Figure 6. The micrograph confirmed that the prepared SLNs were of spherical shape with smooth morphology, nanometric size, narrow size distribution, well dispersed and separated on the surface.

This observation verifies results obtained by a previous study on 5-fluorouracil SLNs where good spherical lipid nanoparticles were performed upon using SLNs with Cetyl palmitate and Glyceryl monostearate [15]. 


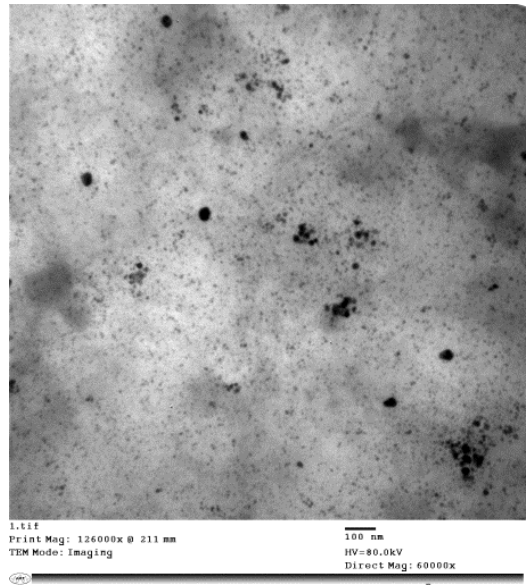

Figure 6. Transmission electron micrograph of RIF loaded SLNs (F4).

\subsection{Pharmacokinetic Study}

Rifampicin concentration in rat plasma after administration of the formulated SLNs dispersion was higher than concentration of the drug suspension and marketed drug suspension at all-time points.

Figure 7 illustrates the enhancement in the pharmacokinetic behavior through plasma concentrationtime curve profiles of RIF upon oral administration of optimized SLNs dispersion (F4) compared to marketed drug suspension and pure drug suspension.

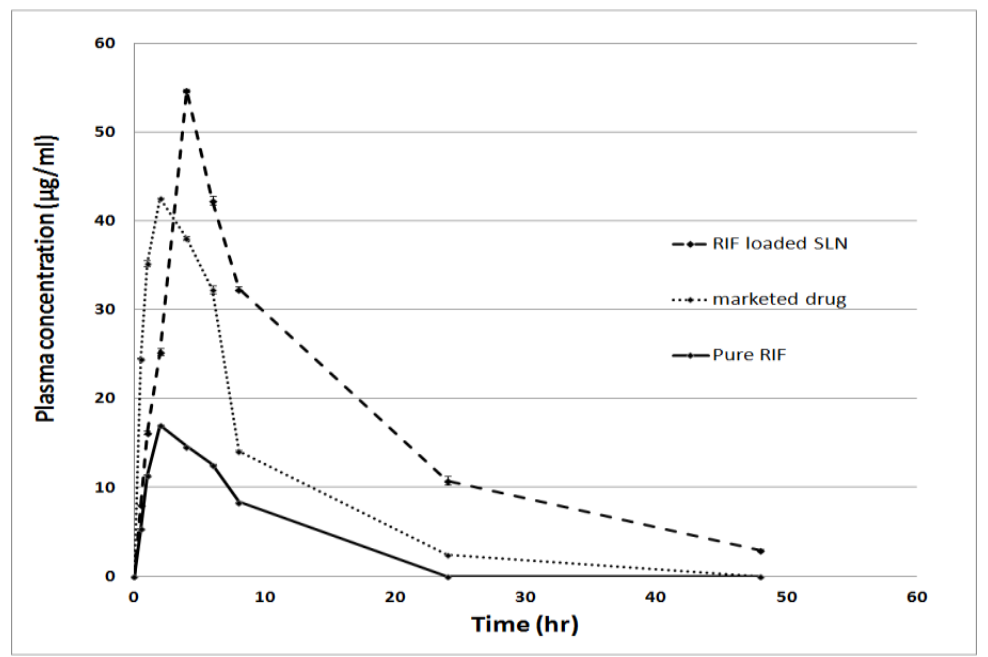

Figure 7. Plasma concentration-time profiles of RIF after oral administration of optimized RIF loaded SLNs dispersion (F4), marketed drug suspension and pure RIF in rats mean $\pm S E(n=3)$.

\subsubsection{Pharmacokinetic Data Analysis}

As shown in Table 2, the maximum concentration of RIF in plasma is $54.66 \mu \mathrm{g} / \mathrm{ml}$ for SLNs dispersion (F4), $42.52 \mu \mathrm{g} / \mathrm{ml}$ for marketed drug suspension and $16.97 \mu \mathrm{g} / \mathrm{ml}$ for pure drug suspension. The Cmax for SLNs dispersion (F4) increased 1.29 and 3.22 folds as compared with marketed drug suspension and pure drug suspension, respectively. Also, SLNs dispersion (F4) shows high area under the curve (974. 1183 $\mu \mathrm{g} . \mathrm{h} / \mathrm{ml})$ when compared with marketed drug (417. $8975 \mu \mathrm{g} . \mathrm{h} / \mathrm{ml})$ and pure drug suspension (166. 1283 $\mu \mathrm{g} . \mathrm{h} / \mathrm{ml}$ ). The relative bioavailability of SLNs dispersion (F4) enhanced 2.33 and 5.86 folds in comparison with marketed drug and pure drug suspension respectively. This indicates that SLNs formula enhanced the solubility and dissolution rate of the drug.

These results indicated improvement in drug absorption for optimized SLNs dispersion (F4) and sustained its release profile when compared with marketed drug and pure drug suspension. The reason is that emulsifiers used in SLNs formulation were able to alter the fluidity of membrane and enhanced drug absorption through the gut. In addition, preparation of RIF as solid lipid nanoparticles not only enhanced its 
solubility but also enhanced its tissue permeability. These results verify observations obtained by a previous study on domperidone SLNs using Tween ${ }^{\circledR} 80$ and Dynasan ${ }^{\circledR} 118$ as solubilizers which showed enhancement in the bioavailability and obtained a sustained release profile of domperidone in comparison with conventional tablet [26].

Reduction of particle size to a nanometer range increased the contact surface area and thus increased the dissolution velocity according to Noyes-Whitney equation [27]. Also, small size of SLNs prolonged drug residence time which improved the drug diffusion [28].

The pharmacokinetic characteristics of the drug loaded SLNs were ordered by the properties of SLNs rather than by the physicochemical characteristics of the drug molecule [29].

Table 2. Pharmacokinetic parameters of RIF following oral administration of optimized SLNs dispersion (F4), marketed drug and pure drug suspension in rats.

\begin{tabular}{lccc}
\hline PK parameters & SLNs dispersion (F4) & Marketed drug & Pure drug suspension \\
\hline Cmax $(\mu \mathrm{g} / \mathrm{ml})$ & 54.66 & 42.52 & 16.97 \\
Tmax $(\mathbf{h})$ & 4 & 2 & 2 \\
AUC0-48h $(\mu \mathrm{g} . \mathrm{h} / \mathrm{ml})$ & 974.1183 & 417.8975 & 166.1283 \\
\hline
\end{tabular}

\section{CONCLUSION}

The suitable choice of excipients (lipid and surfactant) and optimized combination in the SLNs preparation played an important role in enhancing RIF oral absorption and bioavailability. In the current study, RIF loaded SLNs were formulated successfully by a modified micro emulsion-based method. The resultant RIF-SLNs formulae had homogenous particle size and high entrapment efficiency. In vitro release study revealed that the release rate of RIF from all formulae fitted into Higuchi's diffusion model at pH 7.4. In vivo pharmacokinetic study revealed that the relative bioavailability of SLNs dispersion (F4) enhanced 2.33 and 5.86 folds in comparison with marketed drug and pure drug suspension respectively. Based on these results RIF-SLNs could be a successful carrier for the oral delivery of rifampicin.

\section{MATERIALS AND METHODS}

Cetyl palmitate was kindly provided by Gattefosee (Saint-Priest, France). Glyceryl monostearate was purchased from Alpha Chemika (Mumbai, India). Poloxamer 188 was purchased from Caisson laboratories (Utah, United States). Tween ${ }^{\circledR} 80$ was purchased from Elnasr pharmaceutical chemicals (Al Qalyubia, Egypt). Rifampicin was kindly provided by MUP pharmaceutical company (Abusultan, Egypt). Distilled water and freshly prepared reverse osmosis water ( $\mathrm{RO}$ water) were used in all experiments. Dialysis tubing cellulose membrane (molecular weight cut-off 12,000 g/mole) was obtained from Sigma-Aldrich Chemical Company (St.Louis, USA). Ethanol was purchased from PureChem-import (Cairo, Egypt). Sodium chloride was purchased from Elnasr pharmaceutical chemicals (Al Qalyubia, Egypt). Potassium chloride was purchased from Elnasr pharmaceutical chemicals (Al Qalyubia, Egypt). Sodium dihydrogen phosphate was purchased from Elnasr pharmaceutical chemicals (Al Qalyubia, Egypt). Potassium monohydrogen phosphate was purchased from Elnasr pharmaceutical chemicals (Al Qalyubia, Egypt). Methanol and Acetonitrile for HPLC were obtained from Pock S.A. (Gliwice, Poland). All chemicals were used directly as received without further purification.

\subsection{Preparation of RIF loaded SLNs}

A modified micro emulsion-based technique was used for the preparation of SLNs [4]. The lipids (Cetyl palmitate/Glyceryl monostearate) and emulsifiers (Tween ${ }^{\circledR} 80$ /Poloxamer 188) were melted and mixed by magnetic stirring at $80^{\circ} \mathrm{C}$, then $80 \mathrm{mg}$ of Rifampicin was added at $80^{\circ} \mathrm{C}$ and stirred to ensure that all the drug was dispersed. Up to $30 \mathrm{ml}$ of $\mathrm{RO}$ water was added to the mixture at the same temperature. This coarse pre-emulsion was treated with high shear homogenization using an Ultra-Turrax homogenizer (Ultra- Turrax T - 25, IKA, Germany) at 15,000 rpm for 15 minutes, with 30 seconds intervals every two minutes. The resulting microemulsion was immediately dispersed in cold distilled water while stirring $\left(2^{\circ} \mathrm{C}\right.$ to $3^{\circ} \mathrm{C}$ ) with a ratio $1: 2$ for distilled water and pre-emulsion, respectively. The principle of this latter method is to add a warm $\mathrm{O} / \mathrm{W}$ microemulsion into cold water leading to lipid phase precipitation forming fine particles called SLNs. 


\subsection{Measurement of particle size and polydispersity index}

Particle size and polydispersity index of RIF loaded SLNs was determined using Laser Zetameter (Malvern Instruments, ZS). All measurements were done after samples dilution by ultra-purified water to maintain a suitable scattering intensity. DLS data were analyzed at $25^{\circ} \mathrm{C}$ using the general-purpose mode [30].

\subsection{Zeta potential determination}

Zeta potential $(\zeta)$ indicates the surface charge of the nanoparticles. It is used for predicting the stability of nanoparticle suspension and measured in millivolts $(\mathrm{mV})$ [31].

Zeta potential was determined using folded capillary cells by Laser Zetameter (Malvern Instruments, ZS) at $25^{\circ} \mathrm{C}$. Samples were diluted appropriately by RO water to avoid interparticle scattering that can interfere with results.

\subsection{Loading capacity}

The loading capacity (L.C) refers to the percentage amount of the drug that was loaded in the solid lipid nanoparticles according to the bellow equation [32].

$$
\text { L.C }=\frac{\text { Total amount of drug-amount of unloaded drug }}{\text { Nanoparticles weight }} \times 100
$$

\subsection{Encapsulation efficiency}

The encapsulation efficiency percent (E.E \%) refers to the percentage of rifampicin encapsulated in the nanoparticles which were indirectly determined by calculating the concentration of unloaded drug in the dispersion medium using ultracentrifugation. Ultracentrifugation was done using (Hettich centrifuge, Mikro 22R, Germany). Freshly prepared SLNs dispersions $(1 \mathrm{ml})$ were added in the centrifuge tube at 13,000 rpm for $30 \mathrm{~min}$ at $4^{\circ} \mathrm{C}$, the nanoparticles were separated from the medium [32].

The filtrate was then diluted with ethanol up to $10 \mathrm{ml}$, and rifampicin concentration was recorded by spectrophotometry using a calibration curve of rifampicin in ethanol at $337 \mathrm{~nm}$ [33]. All the experiments were processed in triplicates. The encapsulation efficiency was calculated using the below equation.

$$
\mathrm{E} . \mathrm{E}=\frac{\text { Total amount of drug-amount of unloaded drug }}{\text { Total amount of drug }} \times 100
$$

\subsection{In vitro drug release studies}

Drug release studies of RIF loaded SLNs formulae were performed in vitro using a dialysis bag method using 12,000-dalton molecular weight cut-off membrane. The dissolution medium consisted of phosphate buffer saline (PBS) ( $\mathrm{pH}$ 7.4). SLNs dispersion $(1 \mathrm{ml})$ was added in the dialysis bag and was clamped from both ends. The bag was soaked in $20 \mathrm{ml}$ of release medium. To mimic biological conditions, a thermostatic shaker was adjusted at $37 \pm 0.5^{\circ} \mathrm{C}$ and rate 36 cycles per min. At time points $(0,0.5,1,2,4,6,8,24$, $48,72$ and $120 \mathrm{~h}) 1 \mathrm{ml}$ of aliquots from the dissolution medium were withdrawn and replaced by a fresh medium to preserve sink condition. Released drug concentration was measured using a spectrophotometer (Shimadzu UV-1601, Japan) at a maximum wavelength of $337 \mathrm{~nm}$ using a standard calibration curve of RIF. All the experiments were processed in triplicates [4].

\subsection{Differential Scanning Calorimetry (DSC)}

Differential scanning calorimetry thermograms of RIF, Cetyl palmitate, Glyceryl monostearate, their physical mixture and RIF loaded SLNs (F4) were measured using (DSC-50 SHIMADZU, Japan). Approximately $5 \mathrm{mg}$ sample was sealed in an aluminum pan at a heating rate of $10^{\circ} \mathrm{C} / \mathrm{min}$ over a temperature range of $25^{\circ} \mathrm{C}-310^{\circ} \mathrm{C}$ throughout the analysis. The reference pan was left empty and the whole thermal behaviours were measured under nitrogen purge with at a flow rate $30 \mathrm{ml} / \mathrm{min}$. The onset, melting point (peak maximum) and the enthalpy $(\Delta \mathrm{H})$ were recorded [21]. 


\subsection{Fourier Transform Infrared Spectroscopy (FTIR)}

Fourier transform infrared spectra of RIF, Cetyl palmitate, Glyceryl monostearate, their physical mixture and RIF loaded SLNs (F4) were recorded using (FTIR-4100 JASCO, Japan). Solid samples were mixed with (IR) grade potassium bromide then compressed using a hydraulic press to form thin discs. The spectra were recorded at $2 \mathrm{~mm} / \mathrm{s}$ and a resolution of $4 \mathrm{~cm}^{-1}$ within the spectral region of 4000 and $400 \mathrm{~cm}^{-1}$ [21].

\subsection{Transmission Electron Microscopy (TEM)}

Transmission electron microscopy (model JEM-1400, Jeol, Tokyo, Japan) was performed to examine surface morphology and size distribution of RIF loaded SLNs (F4). One drop of a diluted sample solution was added on the surface of carbon-coated copper grid and negatively stained for $30 \mathrm{~s}$ using a drop of aqueous solution of phosphotungestic acid $(2 \% \mathrm{w} / \mathrm{w})$. The grid was tapped with filter paper to remove excess staining solution, leaving aqueous film on the surface. Finally, the grid was dried at $25^{\circ} \mathrm{C}$ for $10 \mathrm{~min}$ before examination [17].

\subsection{Pharmacokinetic Study}

Male Wistar rats (average weight 200 g) were divided into three groups $(n=3)$, group I for administration of pure drug in 1\% sodium carboxymethyl cellulose as suspending agent, group II for administration of marketed RIF suspension (reference group) and group III for administration of optimized RIF loaded SLNs dispersion formula (F4), (test group). They were dosed at $15 \mathrm{mg} / \mathrm{kg}$ via oral gavages. All procedures used in the study were approved by the Institutional Animal Care and Ethical Committee of Suez Canal University [24]. Blood samples $(0.5 \mathrm{ml})$ were collected from each group under light ether anaesthesia from the retro-orbital plexus to heparinised micro centrifuge tubes at different time points. After that, samples were centrifuged at $10000 \mathrm{rpm}$ for $10 \mathrm{~min}$ for plasma separation using (Hettich centrifuge, Mikro 22R, Germany) and samples were stored at $-20^{\circ} \mathrm{C}$ prior to drug analysis [24].

\subsubsection{Determination of RIF Concentration in Rat Plasma Using HPLC (Plasma Sample Analysis)}

This experiment was done using YL9100 HPLC system. Chromatographic separation and quantification were made on reversed phase C-18 column ( $150 \mathrm{~mm} \times 4.6 \mathrm{~mm}$ i.d.j particle size $5 \mu \mathrm{m})$ at room temperature. The mobile phase, consisted of acetonitrile, methanol and water at ratio of 30:5:65 v/v with final $\mathrm{pH} 5.2$, was pumped to the column at a flow rate of $1 \mathrm{ml} / \mathrm{min}$ and the detector was adjusted at wavelength $242 \mathrm{~nm}$ [34]. $1 \mathrm{ml}$ of acetonitrile was added for plasma extraction. Samples were then centrifuged at $5000 \mathrm{rpm}$ for $5 \mathrm{~min}$ and $20 \mu \mathrm{l}$ of the supernatant were filtered and injected directly into the column of the HPLC and peak area values were recorded [35].

Calibration curve was obtained using a concentration range of $50-100 \mu \mathrm{g} / \mathrm{mL}$ of RIF; and showed linearity in between the concentration of RIF and its peak area $\left(R^{2}=0.9864\right)$ [34]. All samples were done in triplicates and mean \pm SD was calculated.

\subsubsection{Pharmacokinetic Data Analysis}

Maximum plasma concentration (Cmax), time to reach $\mathrm{Cmax}(\operatorname{Tmax})$, area under the curve from zero to the sampling time (AUC0-48h ) and half-life (t1/2) are the values obtained from plasma concentrationtime curve [36].

The relative bioavailability (Frel) was calculated to compare the bioavailability of a test formulation with a reference formulation using the following equation:

Frel $=\left[\mathrm{AUC}_{\text {test }} / \mathrm{AUC}\right.$ reference $] \times 100$

[Eq. 3]

Acknowledgements: Authors wished to acknowledge MUP pharmaceutical company (Abusultan, Egypt) for providing rifampicin as gift sample.

Author contributions: Concept - A.G., S.G.; Design - A.G., S.G., Al.G.; Supervision - A.G., S.G.; Resources - A.G., Al.G.; Materials - A.G., Al.G.; Data Collection and/or Processing - A.G., Al.G.; Analysis and/or Interpretation - A.G., Al.G.; Literature Search - A.G., Al.G.; Writing - Al.G.; Critical Reviews - A.G., S.G., Al.G.

Conflict of interest statement: The authors declared no conflict of interest. 


\section{REFERENCES}

[1] Mulla JAS, Hiremath SP, Sharma NK. Repaglinide Loaded Solid Lipid Nanoparticles: Design and Characterization. RGUHS J Pharm Sci. 2012; 2(4): 41-49.

[2] Ebrahimi HA, Javadzadeh Y, Hamidi M, Jalali MB. Repaglinide-Loaded Solid Lipid Nanoparticles: Effect of Using Different Surfactants/Stabilizers on Physicochemical Properties of Nanoparticles. DARU J Pharm Sci. 2015; 23(1): 46. [CrossRef]

[3] Boonme P, Souto EB, Wuttisantikul N, Jongjit T, Pichayakorn W. Influence of Lipids on the Properties of Solid Lipid Nanoparticles from Microemulsion Technique. Eur J Lipid Sci Technol. 2013; 115(7): 820-824. [CrossRef]

[4] Aboutaleb E, Noori M, Gandomi N, Atyabi F, Fazeli MR, Jamalifar H, Dinarvand R. Improved Antimycobacterial Activity of Rifampin Using Solid Lipid Nanoparticles. Int Nano Lett. 2012; 2(1): 33. [CrossRef]

[5] Chokshi NV, Khatri HN, Patel MM. Formulation, Optimization, and Characterization of Rifampicin-Loaded Solid Lipid Nanoparticles for the Treatment of Tuberculosis. Drug Dev Ind Pharm. 2018; 44(12): 1975-1989. [CrossRef]

[6] Shimomura H, Nogami R, Shigeno A, Shimada S, Aoyama T. Influence of Food on Rifampicin Pharmacokinetics in Rats. Biol Pharm Bull. 2016; 39(1): 49-53. [CrossRef]

[7] Mona MA, Amira MM. Solid Lipid Nanoparticles and Nanostructured Lipid Carriers of Tolnaftate: Design, Optimization and In-Vitro Evaluation. Int J Pharm Pharm Sci. 2016; 8(1): 380-385.

[8] Shah B, Khunt D, Bhatt H, Misra M, Padh H. Application of Quality by Design Approach for İntranasal Delivery of Rivastigmine Loaded Solid Lipid Nanoparticles: Effect on Formulation and Characterization Parameters. Eur J Pharm Sci. 2015; 78: 54-66. [CrossRef]

[9] Baek JS, Shin SC, Cho CW. Effect of Lipid on Physicochemical Properties of Solid Lipid Nanoparticle of Paclitaxel. J Pharm Investig. 2012; 42(5): 279-283. [CrossRef]

[10] Golmohammadzadeh S, Mokhtari M, Jaafari MR. Preparation, Characterization and Evaluation of Moisturizing and UV Protecting Effects of Topical Solid Lipid Nanoparticles. Braz J Pharm Sci. 2012; 48(4): 683-690. [CrossRef]

[11] Palie N, Das MK. Preparation and Characterization of Lornoxicam Loaded Solid Lipid Nanoparticles Made from Different Lipids. Int J Pharm Pharm Sci. 2013; 5 Suppl 4: 438-442.

[12] Ding Y. PhD Thesis. Lipid Nanoparticles for Topical Delivery: Solid Lipid Nanoparticles (SLN) \& Smartlipids. Department of Pharmaceutics, the Institute of Pharmacy, Freie University, Berlin, Germany, 2018.

[13] Sharma VK. Solid Lipid Nanoparticles System: an Overview. Int J Res Pharm Sci. 2011; 2(3): 450-461.

[14] Kumar VV, Chandrasekar D, Ramakrishna S, Kishan V, Rao YM, Diwan PV. Development and Evaluation of Nitrendipine Loaded Solid Lipid Nanoparticles: İnfluence of Wax and Glyceride Lipids on Plasma Pharmacokinetics. Int J Pharm. 2007; 335(1-2): 167-175. [CrossRef]

[15] Shenoy VS, Gude RP, Murthy RSR. In Vitro Anticancer Evaluation of 5-Fluorouracil Lipid Nanoparticles Using B16F10 Melanoma Cell Lines. Int Nano Lett. 2013; 3(1): 36. [CrossRef]

[16] Patel M. MSc Thesis. Development, Characterization and Evaluation of Solid Lipid Nanoparticles as a Potential Anticancer Drug Delivery System. College of Pharmacy and Pharmaceutical Science, University of Toledo, Toledo, Ohio, United States, 2012.

[17] Khalil RM, El-Bary AA, Kassem MA, Ghorab MM, Ahmed MB. Solid Lipid Nanoparticles for Topical Delivery of Meloxicam: Development and İn Vitro Characterization. Eur Sci J. 2013; 9 Spec No. 3: 779-798.

[18] Vivek K, Reddy H, Murthy RS. Investigations of the Effect of the Lipid Matrix on Drug Entrapment, In Vitro Release, and Physical Stability of Olanzapine-Loaded Solid Lipid Nanoparticles. AAPS PharmSciTech. 2007; 8(4): 16-24. [CrossRef]

[19] Abdelbary G, Fahmy RH. Diazepam-Loaded Solid Lipid Nanoparticles: Design and Characterization. AAPS PharmSciTech. 2009; 10(1): 211-219. [CrossRef]

[20] Müller RH, Mäder K, Gohla S. Solid Lipid Nanoparticles (SLN) for Controlled Drug Delivery-a Review of the State of the Art. Eur J Pharm Biopharm. 2000; 50(1): 161-177. [CrossRef]

[21] Mohyeldin SM, Mehanna MM, Elgindy NA. The Relevancy of Controlled Nanocrystallization on Rifampicin Characteristics and Cytotoxicity. Int J Nanomedicine. 2016; 11: 2209-2222. [CrossRef]

[22] Paolicelli P, Cerreto F, Cesa S, Feeney M, Corrente F, Marianecci C, Casadei MA. Influence of the Formulation Components on the Properties of the System SLN-Dextran Hydrogel for the Modified Release of Drugs. J Microencapsul. 2009; 26(4): 355-364. [CrossRef] 
[23] Ghaderkhani J, Yousefimashouf R, Arabestani M, Roshanaei G, Asl SS, Abbasalipourkabir R. Improved Antibacterial Function of Rifampicin-Loaded Solid Lipid Nanoparticles on Brucella Abortus. Artif Cells Nanomed Biotechnol. 2019; 47(1): 1181-1193. [CrossRef]

[24] Singh H, Jindal S, Singh M, Sharma G, Kaur IP. Nano-Formulation of Rifampicin with Enhanced Bioavailability: Development, Characterization and İn-Vivo Safety. Int J Pharm. 2015; 485(1-2): 138-151. [CrossRef]

[25] Rahman Z, Zidan AS, Khan MA. Non-Destructive Methods of Characterization of Risperidone Solid Lipid Nanoparticles. Eur J Pharm Biopharm. 2010; 76(1): 127-137. [CrossRef]

[26] Shazly GA, Alshehri S, Ibrahim MA, Tawfeek HM, Razik JA, Hassan YA, Shakeel F. Development of Domperidone Solid Lipid Nanoparticles: In Vitro and İn Vivo Characterization. AAPS PharmSciTech. 2018; 19(4): $1712-1719$. [CrossRef]

[27] Patil H, Feng X, Ye X, Majumdar S, Repka MA. Continuous Production of Fenofibrate Solid Lipid Nanoparticles by Hot-Melt Extrusion Technology: a Systematic Study Based on a Quality by Design Approach. AAPS J. 2015; 17(1): 194-205. [CrossRef]

[28] Stets S, Tavares TM, Peralta-Zamora PG, Pessoa CA, Nagata N. Simultaneous Determination of Rifampicin and İsoniazid in Urine and Pharmaceutical Formulations by Multivariate Visible Spectrophotometry. J Braz Chem Soc. 2013; 24(7): 1198-1205.

[29] Aljaeid BM, Hosny KM. Miconazole-Loaded Solid Lipid Nanoparticles: Formulation and Evaluation of a Novel Formula with High Bioavailability and Antifungal Activity. Int J Nanomedicine. 2016; 11: 441-447. [CrossRef]

[30] Shaker S, Gardouh AR, Ghorab MM. Factors Affecting Liposomes Particle Size Prepared by Ethanol İnjection Method. Res Pharm Sci. 2017; 12(5): 346-352.

[31] Triplett MD. PhD Thesis. Enabling Solid Lipid Nanoparticle Drug Delivery Technology by İnvestigating İmproved Production Techniques. Ohio State University, Columbus, Ohio, United States, 2004.

[32] Ghorab M, Gardouh A, Gad S. Effect of Viscosity, Surfactant Type and Concentration on Physicochemical Properties of Solid Lipid Nanoparticles. Int J Pharm Pharm Sci. 2015; 7(3): 145-153.

[33] Begum A, Basava R, Rao R. Simultaneous Estimation of Rifampicin and İsoniazid in Combined Dosage Form By a Simple UV Spectrophotometric Method. Pharm Lett. 2013; 5(3): 419-426.

[34] Prasanthi B, Ratna JV, Phani RC. Development and Validation of RP-HPLC Method for Simultaneous Estimation of Rifampicin, İsoniazid and Pyrazinamide in Human Plasma. J Anal Chem. 2015; 70(8): 1015-1022. [CrossRef]

[35] Nasr A, Gardouh A, Ghorab M. Novel Solid Self-Nanoemulsifying Drug Delivery System (S-SNEDDS) for Oral Delivery of Olmesartan Medoxomil: Design, Formulation, Pharmacokinetic and Bioavailability Evaluation. Pharmaceutics. 2016; 8(3): 20. [CrossRef]

[36] Akhtar N, Talegaonkar S, Khar RK, Jaggi M. Self-Nanoemulsifying Lipid Carrier System for Enhancement of Oral Bioavailability of Etoposide by P-Glycoprotein Modulation: In Vitro Cell Line and İn Vivo Pharmacokinetic İnvestigation. J Biomed Nanotechnol. 2013; 9(7): 1216-1229. [CrossRef]

This is an open access article which is publicly available on our journal's website under Institutional Repository at http://dspace.marmara.edu.tr. 